\title{
Influences of Funneliformis mosseae on the photosynthetic parameters and active secondary metabolites contents of Astragalus membranaceus and Astragalus membranaceus var. mongholicus
}

\author{
Jiquan Liu ${ }^{\mathrm{a}, *}$, Jin Cai ${ }^{\mathrm{b}}$, Runli He ${ }^{\mathrm{a}}$, Xichun Zhang ${ }^{\mathrm{a}}$ \\ a School of Chinese Drugs, Shanxi University of Chinese Medicine, Yuci 030619, Shanxi, China \\ b Institute of Applied Chemistry, Shanxi University, Taiyuan 030006, Shanxi, China \\ *Corresponding author, e-mail: liujiquan2008@163.com
}

Received 8 Feb 2019

Accepted 5 Jun 2019

\begin{abstract}
Astragalus membranaceus and Astragalus membranaceus var. mongholicus are cultivated in large parts of Shanxi province in China for their high medicinal value in traditional Chinese medicine, and the quality of the medicinal materials is a problem worthy of attention. Traditional strategies to increase quality include use of chemical fertilizer and pesticides, but these may have negative impact on the quality of soil and medicinal materials. In this study, the potential of promoting the quality of the medicinal materials was investigated after inoculating A. membranaceus and A. membranaceus var. mongholicus with mycorrhizal fungus (Funneliformis mosseae). The photosynthetic parameters (net photosynthetic rate, stomatal conductance, transpiration rate and intercellular $\mathrm{CO}_{2}$ concentration) and main active principle contents (astragaloside IV, calycosin-7-glucoside, astragalus polysaccharide and trace element Se) were determined in inoculated and non-inoculated plants. Results showed that there was a positive impact on photosynthetic process and the accumulation of secondary metabolites of the plants inoculated with F. mosseae. Compared with control plants, the photosynthetic parameters of Astragalus plants inoculated with F. mosseae were higher, more active substances (astragaloside IV, calycosin-7-glucoside, astragalus polysaccharide and Se) were found in the roots of inoculated plants. F. mosseae had similar promoting effects on both A. membranaceus and A. membranaceus var. mongholicus. The symbiosis of $F$. mosseae and Astragalus plant would be of benefit to improve the quality of the medicinal materials.
\end{abstract}

KEYWORDS: astragaloside IV, calycosin-7-glucoside, astragalus polysaccharide, Se

\section{INTRODUCTION}

The dried roots of Astragalus membranaceus (Fisch.) Bge. and Astragalus membranaceus (Fisch.) Bge. var. mongholicus (Bge.) Hsiao are commonly used in traditional Chinese medicine for various conditions, and it is reported that astragaloside IV, calycosin-7glucoside, astragalus polysaccharide and trace element Se are the main pharmacodynamic material basis $^{1}$. The Chinese Pharmacopoeia stipulates that the contents of astragaloside IV and calycosin-7glucoside shall not be less than $0.04 \%$ and $0.02 \%$ in both dried roots, respectively. Modern pharmacological research shows that $A$. membranaceus and $A$. membranaceus var. mongholicus have many functions such as regulating human immunity, antiageing, anti-fatigue and anti-tumour effects ${ }^{2,3}$. The dried roots or preparation of these plants are used so frequently in traditional Chinese medicine, that it is said that 8 out of 10 Chinese medicine prescriptions written contain them. There are as many as 200 kinds of Chinese patent drug containing A. membranaceus or A. membranaceus var. mongholi$\mathrm{cus}^{4}$. The plants or herbals are also claimed to have high nutritional value. In 2018, the China Food and Drug Administration listed A. membranaceus and A. membranaceus var. mongholicus as the product of dual use of drug and food ${ }^{5}$. The Chinese market has great demand for A. membranaceus and A. membranaceus var. mongholicus. In international market, the medicinal materials of $A$. membranaceus and A. membranaceus var. mongholicus once topped the list of seven varieties of Chinese herbal medicines, which topped the list of more than $\$ 10$ million in Chinese herbal medicine exports ${ }^{6}$.

It is this strong demand that has nearly depleted the wild resources of $A$. membranaceus and $A$. mem- 
branaceus var. mongholicus in China. Hence the standardized cultivation of $A$. membranaceus and A. membranaceus var. mongholicus is an inevitable choice. At present, the plant product is mainly cultivated by traditional way. According to the theory and literature of Chinese medicine, Shanxi province is the birthplace of $A$. membranaceus and A. membranaceus var. mongholicus. In 2009, a GAP cultivation base for A. membranaceus var. mongholicus was established in Hunyuan county of Shanxi Province, China. By the end of 2017, the planting area of $A$. membranaceus and $A$. membranaceus var. mongholicus reached 35000 ha. However, with the traditional or older cultivation method, the quality of the herbals is unstable due to the non-standard planting site selection and the blind use of chemical fertilizers and pesticides. The use of fertilizers and pesticides also leads to environmental pollution and serious damage to ecological balance ${ }^{7}$. Hence the introduction of new methods and techniques to improve the quality of these herbal products is a subject that needs to be studied in depth.

Arbuscular mycorrhizal fungi (AMF) are a kind of beneficial microorganisms widely distributed in soil. Studies have shown that AMF is a key microorganism in the terrestrial ecosystem and can form a reciprocal symbiosis with more than $90 \%$ of terrestrial plants ${ }^{8}$. Since AMF's mycelium is found in the cortex of the host plant's root system, it can expand the absorption area of the plant's root system, improve the utilization of nutrients and water, improve the photosynthesis of plants, and promote the growth and development of plants ${ }^{9}$. Inoculation of AMF can not only improve the absorption of nutrients in soil by plants, but also improve the resistance of plants to adversity, diseases and pests, and reduce the use of pesticides and the application of chemical fertilizers, which is beneficial to protect the ecological environment while improving the quality of cultivated medicinal materials ${ }^{10}$. It has been found that AMF can strongly stimulate the growth of leguminous plants and the formation of root nodules, and inoculation of AMF can effectively improve the growth potential and quality of cultivated crops, especially the yield and quality of leguminous plants ${ }^{11}$. Inoculation of AMF can lead to abnormal accumulation of secondary metabolites in plants ${ }^{12}$, which are most likely the effective material basis for medicinal plants to prevent and treat diseases. It has been reported that $F$. mosseae is a dominant fungus in the rhizosphere of Astragalus plants ${ }^{13}$.

In this article, by inoculating $F$. mosseae in the rhizosphere of A. membranaceus and A. mem- branaceus var. mongholicus, the influences of F. mosseae on the photosynthetic parameters (net photosynthetic rate, stomatal conductance, transpiration rate and intercellular $\mathrm{CO}_{2}$ concentration) and main active principle contents (astragaloside IV, calycosin-7-glucoside, astragalus polysaccharide and trace element Se) were studied. We try to find a new cultivation method for improving the quality of the medicinal materials.

\section{MATERIALS AND METHODS}

\section{Inoculum of $F$. mosseae}

The inoculum of $F$ mosseae (labelled as BGCHUB01A) was procured from the Institute of Plant Nutrition and Resources, Beijing Academy of Agriculture and Forestry Sciences, China. Every $20 \mathrm{ml}$ of inoculation contains 405 spores.

\section{Reagents}

Reference products of astragaloside IV (110781201616, 98.9\%), calycosin-7-glucoside (111920$201606,98.1 \%)$ and D-anhydrous glucose (110833201707, 99.9\%) were purchased from National Institutes for Food and Drug Control (Beijing, China). Se standard solutions containing $(100 \mathrm{mg} / \mathrm{ml})$ was supplied by Chinese National Standard Material Center. Acetonitrile, methanol, formic acid, $\mathrm{H}_{2} \mathrm{SO}_{4}$, phenol, ethanol, concentrated $\mathrm{HNO}_{3}$ and perchloric acid (Trace metal grade) were purchased from Tianjin Kermel Chemical Testing Co., Ltd., China.

\section{Seedling planting}

The one year old seedlings of $A$. membranaceus (SXTCM-0001509) and A. membranaceus var. mongholicus (SXTCM-0001365) were purchased from Yingxian Qianbao Chinese Herbal Medicine Professional Cooperative, Shanxi province, China. All the seedlings in the trial have the same growth potential and the root length was about $10 \mathrm{~cm}$ of A. membranaceus and $15 \mathrm{~cm}$ of A. membranaceus var. mongholicus, respectively. The experimental site was located in the medicinal plant plantation of Shanxi University of Chinese Medicine.

Before planting, rectangular trenches $(45 \mathrm{~cm}$ long, $15 \mathrm{~cm}$ wide and $15 \mathrm{~cm}$ deep) were dug in an east-west direction (The distance between every two trenches is $30 \mathrm{~cm}$ ). Then a clean nylon cloth (There were round holes with a diameter of $6 \mathrm{~mm}$ on the nylon cloth every $3 \mathrm{~cm}$ ) was spread in each rectangular trench. Every rectangular trench was filled with $10 \mathrm{~cm}$ thick aseptic soil (Soil used for the experiments was a mixture of $30 \mathrm{vol} \%$ sand, 
30 vol \% pine needle humus and 40 vol $\%$ field loamy soil, heated at $105^{\circ} \mathrm{C}$ for $2 \mathrm{~h}$ and allowed to cool down before use). One Astragalus seedling was planted in each trench, and the seedling was laid flat along the east-west direction. The inoculum of $F$. mosseae was spread evenly around the root system at a volume of $20 \mathrm{ml}, 40 \mathrm{ml}$ or $60 \mathrm{ml}$ per seedling. Finally, the seedlings were covered with sterile soil for $5 \mathrm{~cm}$ and routine managements were provided ${ }^{14}$. Seedlings that were not inoculated with inoculum of $F$. mosseae served as controls. Each treatment contains 10 seedlings. All the seedlings were randomly arranged under the same illumination condition. Six months later, the whole roots of two years old were harvested for determination of active substance content.

\section{Determination of photosynthetic parameters}

In July on a sunny day, the net photosynthetic rate, stomatal conductivity, transpiration rate and intercellular $\mathrm{CO}_{2}$ concentration were determined by the LI-6400 portable photosynthesis determination system (LI-COR, USA) from 9:00-11:00. The light intensity was set to $1200 \mu \mathrm{mol} \mathrm{m}^{-2} \mathrm{~s}^{-1}$ and light source was set to red blue light. At the top of each plant, three fully expanded leaves with consistent light were selected for determination ${ }^{15}$. Plants that were not inoculated with inoculum of $F$. mosseae served as controls.

\section{Determination of astragaloside IV}

The roots of all tested plants harvested were dried at $50^{\circ} \mathrm{C}$ and mechanically pulverized to a particle size of approximate $1 \mathrm{~mm}$. Crushed sample (about $4 \mathrm{~g}$ ) was accurately weighed and placed in a round bottom flask. $80 \mathrm{ml}$ of $80 \%$ methanol was added. Sample was extracted by reflux for $4 \mathrm{~h}$. After recovering the solvent, the extract was dissolved with $80 \%$ methanol and fixed in a $10 \mathrm{ml}$ volumetric flask ${ }^{1}$. Reference product of astragaloside IV was dissolved with an appropriate amount of $80 \%$ methanol. All the solutions were filtered with $0.22 \mu \mathrm{m}$ microporous filter membrane for determination.

According to 2015 edition of Chinese Pharmacopoeia $^{1}$, an Agilent 1100 HPLC (Agilent Technologies, Germany), ELSD 2000 Evaporation Detector (Grace Co., Ltd., China) and Dikma C18 column $(4.6 \mathrm{~mm} \times 250 \mathrm{~mm}, 5 \mu \mathrm{m})$ were used for determination of astragaloside IV. The mobile phase was $32 \mathrm{vol} \%$ of acetonitrile +68 vol $\%$ of water. The flow rate was $0.8 \mathrm{ml} / \mathrm{min}$, the injection volume was $20 \mathrm{ml}$, and the column temperature was $30^{\circ} \mathrm{C}$.

\section{Determination of calycosin-7-glucoside}

The preparation method was the same as that of astragaloside IV test solution. Chromatographic method for calycosin-7-glucoside.

According to 2015 edition of Chinese Pharmacopoeia ${ }^{1}$, DAD detector (Agilent Technologies, Germany) was used and the detector was set at the wavelength of $260 \mathrm{~nm}$. The mobile phase was gradient elution (0-20 min, 20-40 vol \% of acetonitrile $+80-60$ vol \% of $0.2 \%$ formic acid solution; 20 $30 \mathrm{~min}, 40 \mathrm{vol} \%$ of acetonitrile $+60 \mathrm{vol} \%$ of $0.2 \%$ formic acid solution). The injection volume was $10 \mathrm{ml}$. Other conditions were the same as those for astragaloside IV determination.

\section{Determination of astragalus polysaccharide}

Crushed sample (about $2 \mathrm{~g}$ ) was accurately weighed and placed in a round bottom flask. $200 \mathrm{ml}$ distilled water was added. Sample was extracted by reflux for $2 \mathrm{~h}$. The extract was fixed with distilled water in a $100 \mathrm{ml}$ volumetric flask. $2 \mathrm{ml}$ of the extraction solution was measured accurately and $10 \mathrm{ml}$ of ethanol was added. The mixed solution was centrifuged for $10 \mathrm{~min}$ at $3000 \mathrm{rpm}$. The precipitate was dissolved in distilled water and fixed to $50 \mathrm{ml}$ for determination ${ }^{6}$. Reference product of D-anhydrous glucose was dissolved with an appropriate amount of distilled water.

The UV-1601 spectrophotometer (Shimadzu Co., Ltd., Japan) and sulphuric acid-phenol method were used to determine the content of astragalus polysaccharide. The measurement wavelength was $490 \mathrm{~nm}$.

\section{Determination of Se}

The roots were rinsed with deionized water to remove dirt and dried in an oven at $50{ }^{\circ} \mathrm{C}$ until constant mass was obtained. The dried samples were finely powdered for determination. All solutions were prepared using deionized water purified by a Milli-Q system (Millipore, Bedford, MA, USA). About $0.2 \mathrm{~g}$ sample was accurately weighed and placed in PTFE digestion vessel. $5 \mathrm{ml}$ mixed acid $\left(\mathrm{HNO}_{3}: \mathrm{HClO}_{4}=4: 1\right)$ were added. The sample and solution were mixed fully and then allowed to stand for $12 \mathrm{~h}$. The vessel was placed in a microwave oven (CEM, Matthews, NC, USA). The temperature was stepped up to $100^{\circ} \mathrm{C}$ in 10 min and maintained for $1 \mathrm{~h}$. Then the temperature was further stepped up to $130^{\circ} \mathrm{C}$ in $5 \mathrm{~min}$ and maintained for $3 \mathrm{~h}$. After cooling, the solution was transferred into a $10 \mathrm{ml}$ polypropylene volumetric tube for determination ${ }^{6}$. 
An ICP-AEMS (Leeman Labs, USA) instrument was used for Se analysis (operational parameters: output power $1.1 \mathrm{~kW}$; frequency $40.68 \mathrm{MHz}$; cooling gas flow $19 \mathrm{l} / \mathrm{min}$; auxiliary air flow $0.6 \mathrm{l} / \mathrm{min}$; atomizer pressure $0.35 \mathrm{MPa}$; peristaltic pump lift rate $1.2 \mathrm{l} / \mathrm{min}$; wavelength $196.026 \mathrm{~nm}$; spectral line level 1 ; detection limit $0.3 \mu \mathrm{g} / \mathrm{l})^{6}$.

\section{Statistical analyses}

Data were subjected to ANOVA using SPSS 18.0. Treatment means were tested with Duncan's Multiple Range. Test at the $5 \%$ level of probability.

\section{RESULTS}

\section{Effects of $F$ mosseae on photosynthetic parameters}

The results of Fig. 1 showed that inoculation of F. mosseae could improve the photosynthetic indexes of the cultivated plants. For A. membranaceus, compared with the control, the three inoculation amounts could significantly increase the values of net photosynthetic rate, stomatal conductance and intercellular $\mathrm{CO}_{2}$ concentration, and the inoculation amounts of $40 \mathrm{ml} /$ plant and $60 \mathrm{ml} /$ plant could significantly promote transpiration rate. For A. membranaceus var. mongholicus, compared with the control, the three inoculation amounts had significant promotion effect on net photosynthetic rate and transpiration rate, and when the inoculation amount was $40 \mathrm{ml} /$ plant and $60 \mathrm{ml} /$ plant, it had significant promotion effect on stomatal conductance and intercellular $\mathrm{CO}_{2}$ concentration.

\section{Effects of $F$. mosseae on astragaloside IV}

Fig. 2 showed that the content of astragaloside IV in roots of $A$. membranaceus and A. membranaceus var. mongholicus gradually increased with the increase of F. mosseae inoculation amount, and the higher the inoculation amount, the higher the content of astragaloside IV. At the same level, the content of astragaloside IV in the root of A. membranaceus var. mongholicus was higher than that in the root of A. membranaceus. For A. membranaceus var. mongholicus, compared with the control, the content of astragaloside IV in root was significantly increased when the inoculation amount was $40 \mathrm{ml} /$ plant and $60 \mathrm{ml} /$ plant. For A. membranaceus, the content of astragaloside IV in root was significantly increased when the inoculation amount was $60 \mathrm{ml} /$ plant.

\section{Effects of $F$. mosseae on calycosin-7-glycoside}

It can be seen in Fig. 3 that the calycosin-7glycoside content in the root of A. membranaceus var. mongholicus was higher than that in the root of A. membranaceus at the same level. Compared with the control, inoculation of $F$ mosseae can significantly increase the calycosin-7-glycoside content in the root of A. membranaceus. When the inoculation amount was $40 \mathrm{ml} /$ plant or $60 \mathrm{ml} /$ plant, the calycosin-7-glycoside content in the root of A. membranaceus var. mongholicus was significantly increased.

\section{Effects of $F$. mosseae on astragalus polysaccharide}

Compared with the control, all treatments can significantly increase the content of astragalus polysaccharide in the roots of $A$. membranaceus and A. membranaceus var. mongholicus. With the increase of inoculation amount, the content of polysaccharide in the roots also significantly increased. There were obvious differences between different inoculation amounts of $F$. mosseae. Inoculating $F$. mosseae had the greatest promotion effect on the content of polysaccharide. At the same level, the polysaccharide content in the root of $A$. membranaceus was higher than that in the root of $A$. membranaceus var. mongholicus (Fig. 4).

\section{Effects of $F$ mosseae on Se}

With the increase of inoculation amount, the content of trace element Se in the roots of A. membranaceus and $A$. membranaceus var. mongholicus was gradually increasing. For A. membranaceus, compared with the control, the content of Se in the root had no significant difference when the inoculation amount was $20 \mathrm{ml} /$ plant, but when the inoculation amount was $40 \mathrm{ml} /$ plant and $60 \mathrm{ml} /$ plant, the enrichment of Se in the root was significantly promoted. For A. membranaceus var. mongholicus, the Se content of all treatments was significantly different from that of the control (Fig. 5).

\section{DISCUSSION}

The photosynthetic parameters (net photosynthetic rate, stomatal conductance, transpiration rate and intercellular $\mathrm{CO}_{2}$ concentration) were important indicators to reflect the exuberance of plant photosynthetic metabolism. The higher the photosynthetic index value, the higher the photosynthetic efficiency of the plant, the more metabolic products will be synthesized ${ }^{16}$. It was reported that inoculation of 

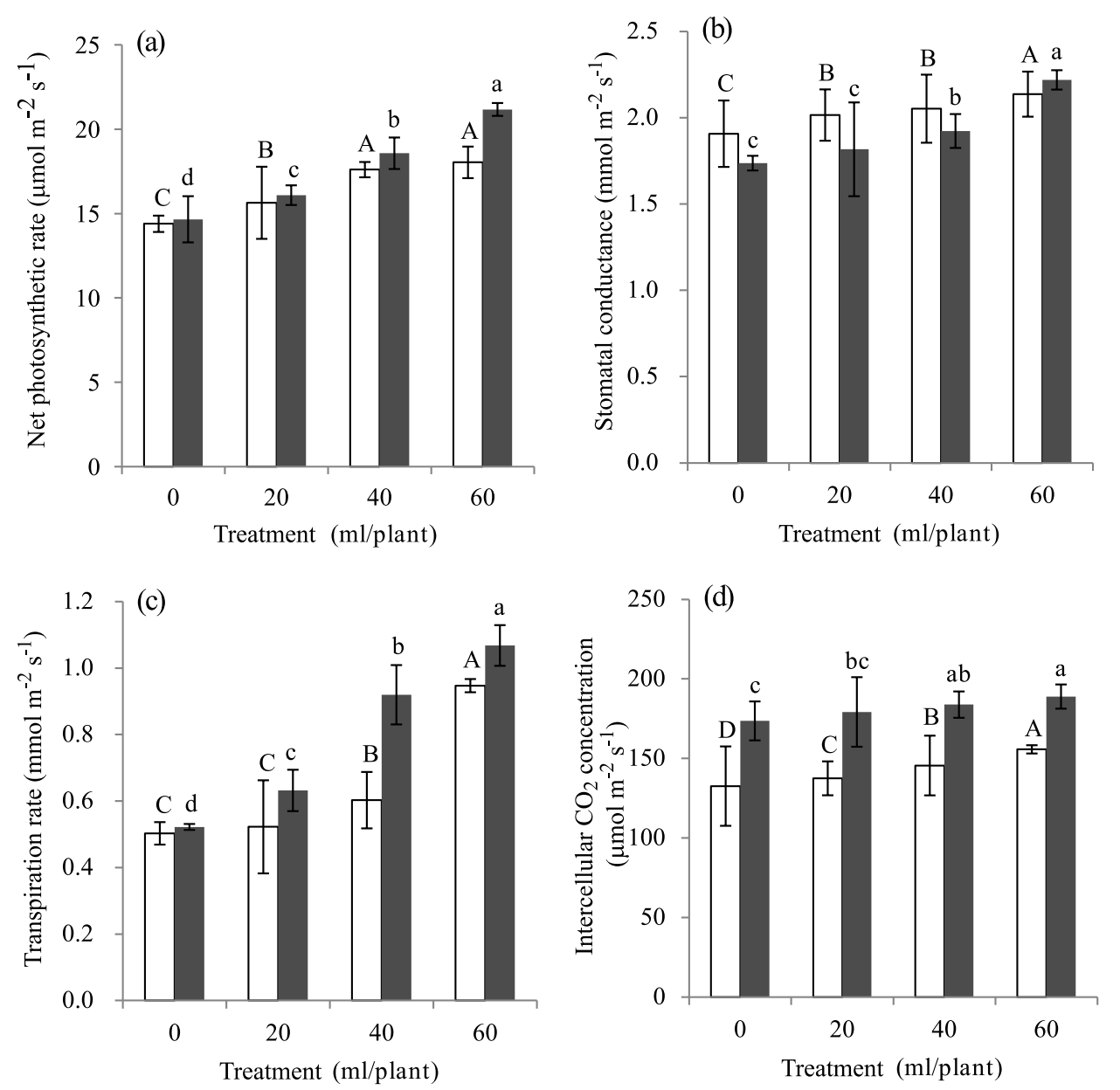

Fig. 1 Effects of F. mosseae on photosynthetic parameters of A. membranaceus and A. membranaceus var. mongholicus: (a) net photosynthetic rate; (b) stomatal conductance; (c) transpiration rate; (d) intercellular $\mathrm{CO}_{2}$ concentration. Different letters indicate a significant difference between the treatment and control (Duncan's Multiple test) of each plant as distinguished by uppercase or lowercase letter.

Glomus mosseae can improve stomatal conductance, transpiration rate and intercellular $\mathrm{CO}_{2}$ concentration of Lolium perenne L. ${ }^{17}$. G. mosseae inoculation can improve the photosynthetic efficiency and flavonoids accumulation of liquorice ${ }^{18}$. In this experiment, the inoculation of $F$ mosseae was beneficial to the photosynthetic parameters of $A$. membranaceus and $A$. membranaceus var. mongholicus. Inoculation amount is clearly positively correlated with promotion effect.

When photosynthetic efficiency increases, plants will synthesize more primary metabolites, such as sugars, lipids, nucleic acids, and proteins. Based on the accumulation of primary metabolites, some important secondary metabolites (such as saponins, flavonoids, and alkaloids) have been further synthesized by using primary metabolites as raw materials or precursors through complex metabolic pathways (such as acetate-malonate pathway, mevalonic acid pathway, cinnamic acid pathway and amino acid pathway) ${ }^{19}$. G. mosseae inoculation can increase the accumulation of the major active components (hinesol, $\beta$-eudesmol and atractylodin) in the rhizome of Atractylodes lancea (Thunb.) DC. ${ }^{20}$ and improved oil content in Mentha arvensis $\mathrm{L}^{21}$. The results of this experiment showed that the inoculation of $A$. membranaceus and A. membranaceus var. mongholicus with $F$. mosseae promoted the accumulation of astragaloside IV, calycosin-7-glucoside, astragalus polysaccharide and Se in roots on the basis of increasing photosynthetic indexes.

AMF are the most widely distributed plant symbionts, and can infect the root systems of over 


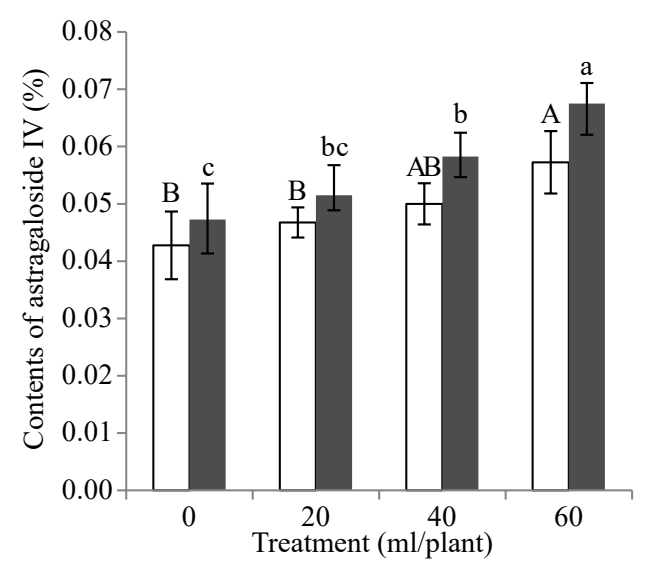

Fig. 2 Effects of $F$. mosseae on astragaloside IV in A. membranaceus and $A$. membranaceus var. mongholicus. Different letters indicate a significant difference between the treatment and control (Duncan's Multiple test) of each plant as distinguished by uppercase or lowercase letter.

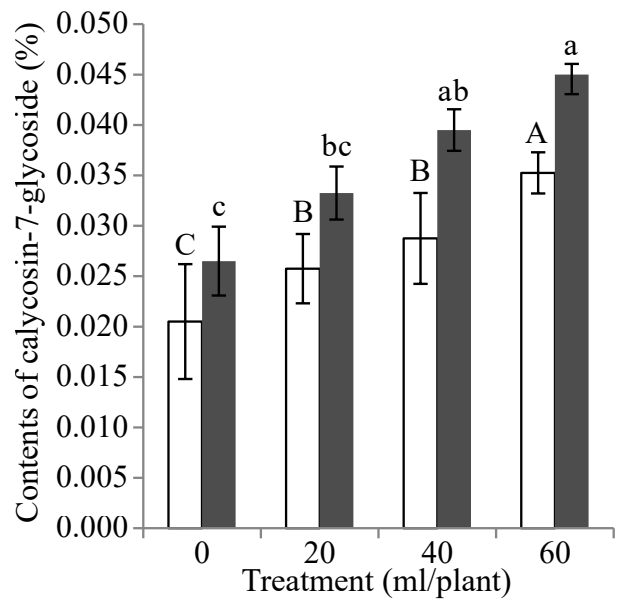

Fig. 3 Effects of $F$. mosseae on calycosin-7-glucoside in A. membranaceus and A. membranaceus var. mongholicus. Different letters indicate a significant difference between the treatment and control (Duncan's Multiple test) of each plant as distinguished by uppercase or lowercase letter.

$80 \%$ of vascular plants ${ }^{22}$. During the symbiotic period of AMF and plants, AMF obtained nutrients by infecting host plant root systems, but also hyphae of AMF can serve as an important channel for soil nutrients entering plants. This greatly improves the absorption of soil nutrients by plant roots $^{23,24}$. Water, minerals and other nutrients needed for plant growth and development can be effectively supplied, especially in the case of poor growth conditions. This channel is particularly important ${ }^{25}$. AMF inoculation can improve the

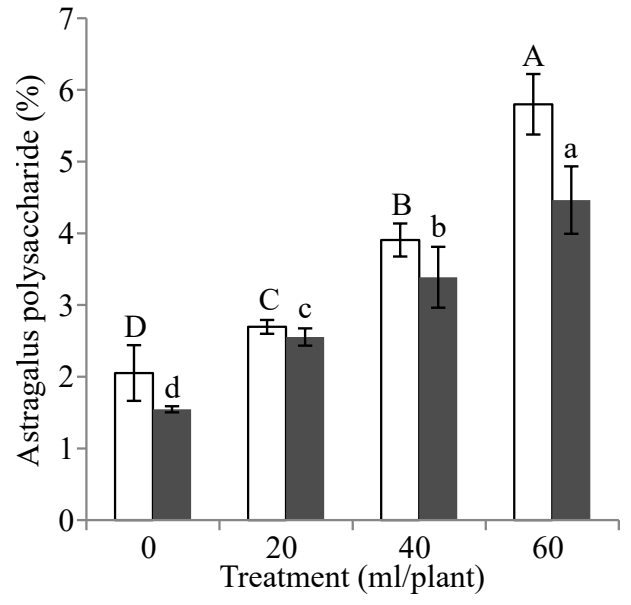

Fig. 4 Effects of $F$. mosseae on astragalus polysaccharide in A. membranaceus and A. membranaceus var. mongholicus. Different letters indicate a significant difference between the treatment and control (Duncan's Multiple test) of each plant as distinguished by uppercase or lowercase letter.

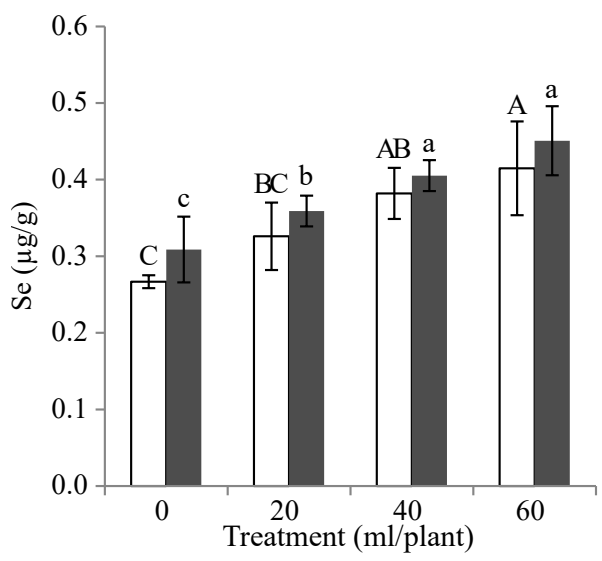

Fig. 5 Effects of F. mosseae on Se in A. membranaceus and $A$. membranaceus var. mongholicus. Different letters indicate a significant difference between the treatment and control (Duncan's Multiple test) of each plant as distinguished by uppercase or lowercase letter.

ability of Astragalus sinicus L. and Zea mays L. to absorb soil nutrients ${ }^{26,27}$. Inoculation of $G$. mosseae can promote the absorption of $\mathrm{Cu}, \mathrm{Zn}$ and other mineral elements by white clover ${ }^{28}$. The formation of plant arbuscular mycorrhizae can promote the absorption of nutrients with low mobility such as $\mathrm{Cu}, \mathrm{Zn}$ and $\mathrm{Fe}$ in soil, and these three elements are often insufficient nutrients in the soil ${ }^{29,30}$. Under drought stress, the relative water content in Vigna radiata $\mathrm{L}$. leaves was $15.3 \%$ higher than that of the control after inoculation with $\mathrm{AMF}^{31}$. 
Water and mineral elements are important factors affecting plant photosynthesis. When the water content of plant leaves is close to water saturation, the leaves can perform normal photosynthesis. When water deficit occurs in plant leaves, stomatal opening decreases or closes, which further affects the diffusion of $\mathrm{CO}_{2}$ into leaf cells. When the water shortage of plant leaves reaches about $20 \%$, photosynthesis is clearly inhibited ${ }^{16}$. The effects of mineral elements on plant photosynthesis are variable. Chlorine and manganese affect the photolysis of water. Iron, copper and phosphorus affect photosynthetic electron transport and photophosphorylation. Potassium, phosphorus and boron promote the transport and transformation of photosynthetic products. Within a certain range, the increase in the absorption of mineral elements will accelerate the photosynthetic rate of plants ${ }^{16}$.

In short, the symbiosis between AMF and host plants expands the channels for host plants to absorb nutrients needed for metabolism and promotes the effective absorption of soil nutrients by host plants. The effective absorption of soil nutrients by the host plant further improves its photosynthetic efficiency, enhances its adaptability to the environment, promotes its growth, and finally improves its yield and quality. This is a virtuous circle.

It was reported that in the pot experiment, when each plant of A. membranaceus was inoculated with $30 \mathrm{~g}$ of $G$. mosseae agent, the root infection rate reached $86.7 \%$. Furthermore, it had a significant promoting effect on plant height, root length, aboveground dry weight and underground dry weight ${ }^{14}$. When a plant of $A$. membranaceus var. mongholicus was inoculated by $10 \mathrm{~g}$ of $G$. mosseae, the root infection rate reached $95 \%$, plant height, root dry weight, stem dry weight, leaf dry weight and number of lateral roots were all higher than the control, the contents of chlorophyll, carotenoid, soluble sugar, $\mathrm{N}$ and $\mathrm{P}$ in the leaves increased, but the inoculation had no significant effect on the content of soluble protein in the leaves ${ }^{32}$.

In this experiment, with inoculating $F$. mosseae, the absorption area of the root system of Astragalus plant was increased, and the transportation of water and mineral elements by the root system of Astragalus plant was accelerated. The satisfaction of water and mineral elements in the metabolism of Astragalus plant will promote the improvement of photosynthesis efficiency and the accumulation of active substances to a certain extent. Within a certain range, the larger the amount of inoculation of $F$. mosseae, the larger the root absorption area and the more mineral elements and water absorbed, the faster the physiological metabolism of Astragalus plant, and the more active substances accumulated. The physiological characteristics of A. membranaceus and A. membranaceus var. mongholicus are different and their light efficiency and light resistance are different during photosynthesis ${ }^{33}$. The infection rates and influence mechanisms of $F$. mosseae on A. membranaceus and A. membranaceus var. mongholicus may be different. The specific mechanism of the effect of $F$ mosseae on Astragalus plant is still unclear and needs to be studied in depth.

In conclusion, F. mosseae could effectively promote the photosynthetic efficiency and the accumulation of active substances of $A$. membranaceus and $A$. membranaceus var. mongholicus. Intensified inoculation of $F$. mosseae will be an effective measure to improve the quality of $A$. membranaceus and A. membranaceus var. mongholicus.

Acknowledgements: This study was supported by National Natural Science Foundation of China (No. 31601677), Natural Science Foundation of Shanxi Province, China (No. 201701D221179; 201701D121180), and Scientific and Technologial Innovation Programs of Higher Education Institutions in Shanxi Province, China (STIP; No. 2016117). We thank Prof Bingchen Liu for his invaluable help with English editing.

\section{REFERENCES}

1. Chinese Pharmacopocia Commission (2015) Pharmacopocia of People's Republic of China, 2015 edn, China Medical Science Press, Beijing. [in Chinese]

2. Guo L, Liu J, Hu Y, Wang D, Li Z, Zhang J, Qin T, Liu X, et al (2012) Astragalus polysaccharide and sulfated epimedium polysaccharide synergistically resist the immunosuppression. Carbohydr Polym 90, 1055-1060.

3. Li R, Chen WC, Wang WP, Tian WY, Zhang XG (2010) Antioxidant activity of Astragalus polysaccharides and anti-tumour activity of the polysaccharides and siRNA. Carbohydr Polym 82, 240-244.

4. Qin XM, Li ZY, Sun HF, Zhang LZ, Zhou R, Feng QJ, Li AP (2013) Status and analysis of Astragali Radix resource in China. Chin J Chin Mater Med 38, 3234-3238. [in Chinese]

5. Yuan H, Zhang SF (2018) Research progress on bioactivity of Astragalus membranaceus and its application in health food. Food Sci 35, 330-334. [in Chinese]

6. Zhou R (2015) Research on Astragalus membranaceus, University Press, Beijing. [in Chinese]

7. Yang ZP, Wang ZH, Fu J, Liu DW, Huang LF (2015) Correlation between chemical constituents 
and ecological factors of Astragalus membranaceus var. mongholicus. Chin J Appl Eco 26, 732-738. [in Chinese]

8. Vergeer P, Berg LTL, Baar J, Quborg NJ, Roelofs JGM (2006) The effect of curf cutting on plant and arbuscular mycorrhizal apore recolonisation: Implications for heathland restoration. Biol Conserv 129, 226-235.

9. Cho K, Toler H, Lee J, Ownley B, Stutz JC, Moore JL, Augé RM (2006) Mycorrhizal symbiosis and response of sorghum plants to combined drought and salinity stresses. J Plant Physiol 163, 517-528.

10. Zeng Y, Guo LP, Huang LQ, Zhou J, Sun YZ (2007) AMF and its application in TCM cultivation. World Sci Tech 9, 83-87. [in Chinese]

11. Crush JR (1974) Plant growth responses to vesculararbuscular mycorrhize VII. Growth and nodulation of some herbage legumes. New Phytol 73, 743-749.

12. Morandi D (1996) Occurrence of phytoalexins and phenolic compounds in endomycorrhizal interactions, and their potential role in biological control. Plant Soil 185, 241-251.

13. Wang YS, Zhang SB, Zhang MQ (2012) Mycorrhizal Resources and Germplasm Resources of Chinese Arbuscular mycorrhizal Fungi, China Agricultural Publishing House, Beijing. [in Chinese]

14. He XL, Liu T, Zhao LL (2009) Effects of inoculating AM fungi on physiological characters and nutritional components of Astragalus membranaceus under different $\mathrm{N}$ application levels. Chin J Appl Ecol 20, 2118-2122.

15. Ma RL, Xu SR, Chen Y, Jing YM, Li CD (2018) Effects of drought stress on photosynthetic characteristics and chlorophyll fluorescence parameters of Astragalus leaves. $J$ Chin Med Mater 41, 531-534.

16. Zhou YL (2000) Plant Biology, Higher education press, Beijing. [in Chinese]

17. Tian $\mathrm{Y}$, Zhang $\mathrm{HH}$, Meng $\mathrm{XY}$, Wang J, Hu JW, Sun GY (2013) Effects of arbuscular mycorrhizal fungi (Glomus mosseae) on growth and photosynthesis characteristics of Lolium perenne L. under Cd contaminationed soil. Acta agrestia sinica 21, 135-141. [in Chinese]

18. Chen M, Yang G, Sheng Y, Li PY, Qiu HY, Zhou XT (2017) Glomus mosseae inoculation improves the root system architecture, photosynthetic efficiency and flavonoids accumulation of liquorice under nutrient stress. Front Plant Sci 8, 931.

19. Liu CS (2016) Pharmaceutical Botany, 10th edn, China Traditional Chinese Medicine Publishing House, Beijing. [in Chinese]

20. Liang XF, Tang MJ, Lv LX, Zhao XY, Dai CC (2018) Effects of three arbuscular mycorrhizal fungi (AMF) species on the growth, physiology, and major components of essential oil of Atractylodes lancea (Thunb.) DC. Chin J Eco 37, 1871-1879. [in Chinese]
21. Bharti N, Baghel S, Barnawal D, Yadav A, Kalra A (2013) The greater effectiveness of Glomus mosseae and Glomus intraradices in improving productivity, oil content and tolerance of salt-stressed mentholmint (Mentha arvensis). J Sci Food Agric 93, 2154-2161.

22. Li S (1998) Different arbuscular mycorrhizal fungal species are potential determinants of plant community structure. Ecology 79, 2082-91.

23. Karagiannidis N, Bletsos F, Stavropoulos N (2002) Effect of Verticillium wilt (Verticillium dahlia Kleb.) and mycorrhiza (Glomus mosseae) on root colonization, growth and nutrient uptake in tomato and eggplant seedlings. Sci Hortic 94, 145-156.

24. Graham JH, Linderman RG, Menge JA (1982) Development of external hyphae by different isolates of mycorrhizal Glomus spp. in relation to root colonization and growth of troger citrange. New Phytol 91, 183-189.

25. Wu QS (2015) Arbuscular mycorrhizal Research and Application of Horticultural Plants, China Science Press, Beijing. [in Chinese]

26. Chen XH, Zhao B (2009) Arbuscular mycorrhizal fungi mediated uptake of nutrient elements by Chinese milk vetch (Astragalus sinicus L.) grown in lanthanum spiked soil. Biol Fertil Soils 45, 675-678.

27. Zhang $\mathrm{HH}$, Tang $\mathrm{M}$, Chen $\mathrm{H}$, Zheng CL, Niu ZC (2010) Effect of inoculation with AM fungi on lead uptake, translocation and stress alleviation of Zea mays L. seedlings planting in soil with increasing lead concentrations. Eur J Soil Biol 46, 306-311.

28. Tarafdar JC (1995) Effect of vasicular-arbuscular mycorrhizal and phosphatase-producing fungal inoculation on growth and nutrition of white clover supplied with organic phosphorus. Folia Microbiol 40, 327-332.

29. Kothari SK, Marschner H, Romheld V (1991) Effect of a vesicular arbuscular mycorrhizal fungus and micro-organisms on manganese reduction in the rhizosphere and manganese concentrations in maize (Zea mays L.). New Phytol 117, 649-655.

30. Liu A, Hamel C, Hamilton RI, Ma BL, Smith DL (2000) Acquisition of Cu, Zn, Mn and Fe by mycorrhizal maize (Zea mays L.) grown in soil at different P and micronutrient levels. Mycorrhiza 9, 331-336.

31. Liu RJ, Hao WY (1994) Effects of AM mycorrhizal fungi on plant water metabolism. Acta Pedologica Sinica 31, 46-53. [in Chinese]

32. Shi L (2007) Ecological and physiological studies of AM fungi from Astragalus membranaceus var. mongholicus. MA.Sc thesis, Hebei University, China.

33. Song N,Guo PY, Yuan XY (2011) Light use, heat dissipation and light patience of Astragalus membranaceus and A. membranaceus var. mongholicus seedlings. Acta Bot Boreal-Occident Sin 31, 2283-2289. [in Chinese] 